\title{
Incidence and Risk Factors of Symptomatic Hiatal Hernia Following Resection for Gastric and Esophageal Cancer
}

\author{
ANDREAS ANDREOU ${ }^{1,2}$, SINA PESTHY $^{1}$, BENJAMIN STRUECKER ${ }^{1,3}$, MEHRAN DADRAS $^{1}$, \\ JONAS RAAKOW ${ }^{1}$, SEBASTIAN KNITTER ${ }^{1}$, GREGOR DUWE ${ }^{1}$, IGOR M. SAUER ${ }^{1}$, ANIKA SOPHIE BEIERLE ${ }^{1}$, \\ CHRISTIAN DENECKE ${ }^{1}$, SASCHA CHOPRA ${ }^{1}$, JOHANN PRATSCHKE ${ }^{1}$ and MATTHIAS BIEBL ${ }^{1}$ \\ ${ }^{1}$ Department of Surgery, Campus Charité Mitte and Campus Virchow Klinikum, \\ Charité - Universitätsmedizin Berlin, Berlin, Germany; \\ ${ }^{2}$ Berlin School of Integrative Oncology (BSIO), Berlin, Germany; \\ ${ }^{3}$ Berlin Institute of Health (BIH), Berlin, Germany
}

\begin{abstract}
Background/Aim: Symptomatic hiatal hernia (HH) following resection for gastric or esophageal cancer is a potentially life-threatening event that may lead to emergent surgery. However, the incidence and risk factors of this complication remain unclear. Patients and Methods: Data of patients who underwent resection for gastric or esophageal cancer between 2005 and 2012 were assessed and the incidence of symptomatic $H H$ was evaluated. Factors associated with an increased risk for HH were investigated. Results: Resection of gastric or esophageal cancer was performed in 471 patients. The primary tumor was located in the stomach, cardia and esophagus in $36 \%, 24 \%$, and $40 \%$ of patients, respectively. The incidence of symptomatic $H H$ was $2.8 \%(n=13)$. All patients underwent surgical hernia repair, 8 patients $(61.5 \%)$ required emergent procedure, and 3 patients (23\%) underwent bowel resection. Morbidity and mortality after $\mathrm{HH}$ repair was $38 \%$ and $8 \%$, respectively. Factors associated with increased risk for symptomatic HH included Body-Mass-Index (median BMI with HH 27 (23-35) vs. BMI without HH 25 (15-51), p=0.043), diabetes (HH rate: with diabetes, $6.3 \%$ vs. without diabetes, $2 \%, p=0.034$ ), tumor location (HH rate: stomach, $1.2 \% \mathrm{vs.}$ esophagus, $1.1 \%$ vs. cardia, $7.9 \%, p=0.001)$, and resection type (HH rate: total/subtotal gastrectomy, $0.7 \%$ vs. transthoracic esophagectomy, $2.7 \%$ vs. extended gastrectomy, $6.1 \%$, p=0.038). Conclusion: $H H$ is a major adverse event after resection for gastric or esophageal cancer especially among patients undergoing extended gastrectomy for cardia cancer
\end{abstract}

Correspondence to: Dr. Andreas Andreou, Department of Surgery, Campus Charité Mitte and Campus Virchow Klinikum, Charité Universitätsmedizin Berlin, Augustenburger Platz 1, 13353 Berlin, Germany. Tel: +49 30450652274, Fax: +49 30450552900, e-mail: andreas.andreou@charite.de

Key Words: Hiatal hernia, gastric cancer, esophageal cancer. requiring a high rate of repeat surgery. Therefore, intensive follow-up examinations for high-risk patients and early diagnosis of asymptomatic patients are essential for selecting patients for elective surgery to avoid unpredictable emergent events with high morbidity and mortality.

Esophageal and gastric cancer are reported as leading causes of cancer-related deaths with rapidly increasing incidence. Worldwide, more than 450,000 people are affected by tumors located in the esophagus (1). Gastric cancer is described as the fifth most common type of cancer in the world (2). Despite advances in endoscopic and systemic therapy, oncological resection in the form of esophagectomy and gastrectomy remain the mainstay of treatment for these serious malignancies of the digestive system $(3,4)$. In recent studies, morbid postoperative complications, such as de novo hiatal hernia $(\mathrm{HH})$ have attracted attention among surgeons and oncologists. Diaphragmatic herniation following esophagectomy has been the subject of current reports in medical literature with an estimated incidence of $0.4-19.4 \%$ (5-15). Gastrectomy accompanied by extensive hiatal dissection is also correlated with hernia formation postoperatively (16). The incidence of $\mathrm{HH}$ after gastrectomy ranges from $0.19-47 \%$ (17-26).

Even though postoperative $\mathrm{HH}$ following esophagectomy and gastrectomy is still considered a rare complication, $\mathrm{HH}$ may be a potentially life-threatening event that remarkably deteriorates the postoperative and long-term outcome of patients. Operative hernia repair is recommended (27), especially in case of symptomatic herniation or a progressive increase in size of HH (9). More importantly, HH may also present with grievous complications, such as severe respiratory compromise, intestinal ischemia with perforation (16), bowel obstruction, and strangulation (27) leading to emergent surgery.

However, studies in which risk factors for $\mathrm{HH}$ have been accurately investigated are lacking. Thus, the aim of this study was to evaluate the incidence of symptomatic $\mathrm{HH}$ in 
patients who underwent resection for gastric or esophageal cancer with curative intent and identify factors associated with an increased risk for the development of this potentially life-threatening complication.

\section{Materials and Methods}

Patient inclusion criteria. Following approval from the institutional review board (EA4/052/14), a retrospective review of clinicopathological data of all consecutive patients treated with curative resection for gastric and esophageal cancer between 2005 and 2012 at the Charité, Campus Virchow-Klinikum in Berlin, Germany was conducted. Patients with stage IV disease and those who underwent palliative resection were excluded from the database. Patients undergoing surgery for benign disease were also excluded from the analysis.

Esophageal resections during this period were predominantly performed using a transthoracic approach and a gastric pull up as previously reported (28). Anastomotic reconstruction after gastric resection included a Roux-en-Y-esophagojejunostomy (28).

Surgical management of the esophageal hiatus. During the initial operation, the hiatus was first dissected and in cases of esophageal resection or transhiatally extended gastric resection, the diaphragm was opened strictly anteriorly up to the left inferior diaphragmatic vein, which was transsected during esophageal resection. Both diaphragmatic crurae were preserved. After mediastinal lymphadenectomy and reconstruction, the hiatus was not closed on a routine basis.

Diagnosis and perioperative management of $\mathrm{HH}$. For the purpose of this study, $\mathrm{HH}$ was defined as an anatomic defect of the hiatus diaphragmaticus following esophagectomy or gastrectomy that allowed elements of the abdominal cavity to herniate into the thorax, usually associated with a striking left-sided predominance (5-7).

Patients were included in the study if they presented with $\mathrm{HH}$ related symptoms making surgical intervention necessary. For the diagnosis of $\mathrm{HH}$ and preoperative assessment of the patients, medical history, physical examination, serum laboratory tests, computed tomography (CT) with contrast agent of the chest and the abdomen, and an anesthesia evaluation were performed.

The extent of the surgery depended on whether the procedure was elective or emergent as well as from the intraoperative findings including ischemia of the herniated intestine and the need for bowel resection. In general, after reduction of the herniated bowel, the crurae were approximated posteriorly by non-resorbable sutures, if possible. Mesh augmentation was used if the hiatus could be closed upon surgeon's discretion. If the hiatus could not be reapproximated, in cases of a retrocolic Roux-Y esophagojejunostomy, the transverse mesocolon was used as a "curtain" in front of the hiatus and the alimentary jejunal loop was straightened between the anastomosis and the mesocolon and sewed to the mesocolon. In cases of esophageal resection and a large diaphragmatic defect, which could not be closed, a non-resorbable mesh was used as an inlay patch to the diaphragm anterior to the gastric conduit.

In case of emergency surgery, patients were postoperatively admitted to a specialized surgical intensive care unit and were closely observed for postoperative complications including intraabdominal infection, bowel ischemia, wound infection, pneumonia, pleural effusions, and organ failure. Postoperative morbidity was defined as any complication within 90 days and postoperative mortality was defined as any in-hospital death after surgery.
Statistical analysis. The primary endpoint of this study was to evaluate the incidence of symptomatic $\mathrm{HH}$ requiring surgical treatment, and identify risk factors associated with an increased risk for $\mathrm{HH}$. Quantitative and qualitative variables were expressed as medians (range) and frequencies. Chi-squared or Fisher's exact test and the Mann-Whitney $U$-test were used to compare categorical and continuous variables, as appropriate. To identify factors associated with an increased risk for $\mathrm{HH}$ following resection for gastric and esophageal cancer, we evaluated the following clinicopathologic variables: sex (male $v s$. female), median age at resection in years, median body-mass-index (BMI), tumor location (esophagus $v s$. cardia $v s$. stomach), resection type (transthoracic esophagectomy $v s$. extended gastrectomy $v s$. total/subtotal gastrectomy), the presence of diabetes (yes $v s$. no), the presence of cardiovascular disease (yes $v s$. no), the presence of pulmonary disease (yes vs. no), the presence of liver cirrhosis (yes $v s$. no), the presence of renal insufficiency (yes vs. no), American Society of Anesthesiologists (ASA) physical status (I $v s$. II $v s$. III $v s$. IV), the use of preoperative chemotherapy (yes $v s$. no), the use of preoperative radiotherapy (yes $v s$. no), median duration of resection time in minutes, the need for intraoperative transfusions (yes vs. no), T stage (T1 vs. T2 vs. T3 $v s$. T4), N stage (N0 vs. N1 vs. N2 vs. N3), Union for International Cancer Control (UICC) stage (I vs. II $v s$. III), presence of lymphangiosis carcinomatosa (yes vs. no), presence of venous invasion (yes $v s$. no), tumor differentiation (G3vs. G1/2), resection margins (R1 vs. R0), histologic type of tumor (adenocarcinoma $v s$. squamous cell carcinoma), presence of anastomotic leak (yes $v s$. no), the use of postoperative chemotherapy (yes $v s$. no), and the use of postoperative radiotherapy (yes $v s$. no).

$p$-Values $<0.05$ were considered statistically significant. Statistical analyses were performed using the SPSS software package, version 22 (IBM, Armonk, NY).

\section{Results}

Patient characteristics. During the study period, a total of 471 esophageal and gastric resections for malignant disease were performed. The clinicopathological data of these patients are summarized in Table I. Thirteen patients suffered symptomatic HH. All of them were men and the median age was 63 (range $=39-78$ years) years. Among patients with symptomatic $\mathrm{HH}$, the primary tumor was located in stomach, cardia, and esophagus in $17 \%, 69 \%$, and $16 \%$ of patients, respectively. Total or subtotal gastrectomy, extended gastrectomy, and transthoracic esophagectomy was performed in 32\%, 20\%, and $48 \%$ of patients who developed $\mathrm{HH}$, respectively.

Incidence of symptomatic $H H$ and clinical presentation. After a median follow-up time of 35 months, the incidence of symptomatic hiatal hernia following esophageal and gastric resection for carcinoma was $2.8 \%(n=13)$ (Table II) and the median time between oncologic resection and diagnosis of the $\mathrm{HH}$ was 15 (range=0.1-57 months) months. With regards to the tumor location, $1.1 \%$ of patients with esophageal cancer $(n=2), 7.9 \%$ of patients with cardia cancer $(n=9)$, and $1.2 \%$ of patients with gastric cancer $(n=2)$ developed symptomatic de novo $\mathrm{HH}$ requiring surgical 
Table I. Clinicopathological characteristics of 471 patients who underwent resection for gastric or esophageal carcinoma.

\begin{tabular}{|c|c|c|c|c|}
\hline Characteristics & $\begin{array}{l}\text { All Patients } \\
(\mathrm{N}=471)\end{array}$ & $\begin{array}{c}\text { No Hiatal Hernia } \\
\qquad(\mathrm{N}=458)\end{array}$ & $\begin{array}{l}\text { Hiatal Hernia } \\
\qquad(\mathrm{N}=13)\end{array}$ & $p$-Value* \\
\hline Male gender, $\%$ & 75 & 74 & 100 & 0.035 \\
\hline Median age at resection (range), years & $65(28-89)$ & $65(28-89)$ & $63(39-78)$ & 0.937 \\
\hline Median BMI (range) & $25(15-51)$ & $25(15-51)$ & $27(23-35)$ & 0.043 \\
\hline Tumor location, $\%$ & & & & 0.001 \\
\hline Esophagus & 40 & 40 & 16 & \\
\hline Cardia & 24 & 23 & 69 & \\
\hline Stomach & 36 & 37 & 15 & \\
\hline Resection Type, \% & & & & 0.038 \\
\hline Transthoracic esophagectomy & 48 & 48 & 46 & \\
\hline Extended gastrectomy & 20 & 20 & 46 & \\
\hline Total/subtotal gastrectomy & 32 & 32 & 8 & \\
\hline Diabetes, $\%$ & 16 & 16 & 39 & 0.034 \\
\hline Cardiovascular disease, $\%$ & 57 & 57 & 62 & 0.720 \\
\hline Pulmonary disease, $\%$ & 15 & 16 & 8 & 0.440 \\
\hline Liver cirrhosis, $\%$ & 2 & 2 & 0 & 0.572 \\
\hline Renal insufficiency, $\%$ & 8 & 8 & 0 & 0.279 \\
\hline ASA physical status, $\%$ & & & & 0.562 \\
\hline I & 4 & 4 & 8 & \\
\hline II & 40 & 41 & 25 & \\
\hline III & 54 & 53 & 67 & \\
\hline IV & 2 & 2 & 0 & \\
\hline Preoperative chemotherapy, $\%$ & 53 & 52 & 77 & 0.081 \\
\hline Preoperative radiotherapy, $\%$ & 14 & 14 & 8 & 0.517 \\
\hline Median duration of resection (range), min & $288(96-687)$ & $288(96-687)$ & $292(195-389)$ & 0.856 \\
\hline Need for intraoperative transfusions, $\%$ & 21 & 21 & 8 & 0.290 \\
\hline T Stage, $\%$ & & & & 0.597 \\
\hline $\mathrm{T} 1$ & 20 & 21 & 8 & \\
\hline $\mathrm{T} 2$ & 17 & 17 & 23 & \\
\hline $\mathrm{T} 3$ & 60 & 59 & 69 & \\
\hline $\mathrm{T} 4$ & 3 & 3 & 0 & \\
\hline N Stage, $\%$ & & & & 0.364 \\
\hline No & 54 & 54 & 69 & \\
\hline N1 & 17 & 17 & 23 & \\
\hline $\mathrm{N} 2$ & 15 & 15 & 0 & \\
\hline N3 & 14 & 14 & 8 & \\
\hline UICC Stage, $\%$ & & & & 0.203 \\
\hline I & 24 & 25 & 23 & \\
\hline II & 48 & 47 & 69 & \\
\hline III & 28 & 28 & 8 & \\
\hline Lymphangiosis carcinomatosa, $\%$ & 31 & 31 & 31 & 0.986 \\
\hline Venous invasion, $\%$ & 12 & 11 & 15 & 0.653 \\
\hline Poorly differentiated carcinoma (G3), \% & 49 & 48 & 73 & 0.104 \\
\hline Positive resection margins (R1), \% & 8 & 8 & 8 & 0.982 \\
\hline Histologic type, $\%$ & & & & 0.088 \\
\hline Adenocarcinoma & 82 & 82 & 100 & \\
\hline Squamous cell carcinoma & 18 & 18 & 0 & \\
\hline Anastomotic leak, $\%$ & 9 & 9 & 0 & \\
\hline Postoperative chemotherapy, $\%$ & 37 & 37 & 62 & 0.067 \\
\hline Postoperative radiotherapy, $\%$ & 7 & 7 & 8 & 0.882 \\
\hline
\end{tabular}

*Comparison of patients with and without hiatal hernia. BMI: Body-mass-index; UICC: Union for International Cancer Control; ASA: American Society of Anesthesiologists.

treatment (Table II). Abdominal pain $(n=8,62 \%)$ was the most common presenting symptom, followed by emesis $(\mathrm{n}=6,46 \%)$, and bowel obstruction $(\mathrm{n}=6,46 \%)$ (Table III).
Surgical management of patients with symptomatic $H H$ and postoperative results. All patients underwent open operative hernia repair including 8 patients $(62 \%)$ who required an 
Table II. Incidence of hiatal hernia $(\mathrm{HH})$ according to the location of the resected tumor.

\begin{tabular}{lcc}
\hline Tumor location & Number of HH (n) & Incidence of HH (\%) \\
\hline Esophagus $(\mathrm{n}=171)$ & 2 & 1.1 \\
Cardia $(\mathrm{n}=114)$ & 9 & 7.9 \\
Stomach $(\mathrm{n}=186)$ & 2 & 1.2 \\
\hline Total $(\mathrm{n}=471)$ & 13 & 2.8 \\
\hline
\end{tabular}

emergent procedure, and 3 patients (23\%) who underwent resection of ischemic herniated bowel (Table III). Primary repair of the HH was performed by re-approximating the diaphragmatic crura with $(n=3)$ or without $(n=10)$ the use of a mesh implant.

The in-hospital mortality rate was $8 \%(\mathrm{n}=1)$. Postoperative death was related to myocardial infraction following emergency surgery for acute mechanical ileus due to incarcerated and torqued small bowel in the thoracic cavity. The 64-year-old patient underwent resection of the ischemic intestinal segments and developed postoperative sepsis requiring admission in the surgical intensive care unit. Postoperative 90-day morbidity was $38 \%(\mathrm{n}=5)$ and included pulmonary complications (pneumonia, pneumothorax, pleura effusions), bleeding complications, and infectious complications in 3,1 , and 1 patient, respectively.

Prognostic factors for symptomatic HH. Factors associated with an increased risk for the development of symptomatic $\mathrm{HH}$ are summarized in Table I and included Body-MassIndex (median BMI with HH 27 (23-35) vs. BMI without HH 25 (15-51), $p=0.043$ ), diabetes (HH rate: with diabetes, $6.3 \% v s$. without diabetes, $2 \%, p=0.034)$, tumor location (HH rate: stomach, $1.2 \% v s$. esophagus, $1.1 \% v s$. cardia, $7.9 \%, p=0.001$ ), and resection type (HH rate: total/subtotal gastrectomy, $0.7 \% v s$. transthoracic esophagectomy, $2.7 \% v s$. extended gastrectomy, $6.1 \%, p=0.038$ ).

\section{Discussion}

In the past decade, several studies have reported the development of $\mathrm{HH}$ following resection for gastric or esophageal cancer $(9,29)$. However, factors associated with an increased risk for symptomatic de novo $\mathrm{HH}$ remain unclear and recommendations for the management of patients undergoing resection for gastric or esophageal cancer are still not established. Our current study examined factors associated with symptomatic de novo $\mathrm{HH}$ and indicated that patients with tumors located in the cardia requiring extended gastrectomy have a significant risk for this complication. Diabetes and obesity were additional considerable risk factors.
Table III. Perioperative characteristics of 13 patients who underwent repair of hiatal hernia $(n=13)$.

\begin{tabular}{lc}
\hline Characteristics & Number of patients (\%) \\
\hline Emesis & $6(46 \%)$ \\
Abdominal pain & $8(62 \%)$ \\
Bowel obstruction & $6(46 \%)$ \\
Emergent procedure & $8(62 \%)$ \\
Bowel resection & $3(23 \%)$ \\
Postoperative morbidity & $5(38 \%)$ \\
Postoperative mortality & $1(8 \%)$ \\
\hline
\end{tabular}

Iatrogenic alterations of the upper GI anatomy, such as enlargement of the hiatus during esophagectomy was the only investigated risk factor in previous analyses that has been considered to be significantly associated with postoperative herniation $(9,29)$. Van Sandwick et al. have postulated already in 1999, that the main cause of HH may be the extensive blunt dissection of the hiatus (29). A series published in 2011 hypothesized that $\mathrm{HH}$ is a result of increased intraabdominal pressure and suction effect of the negative intrathoracic pressure leading to progressive hiatal dilation $(5,6)$.

Price et al. presented their results on the incidence of diaphragmatic hernia following minimally invasive esophagectomy (6). Laparoscopic surgery is generally known to decrease the extent of postoperative peritoneal adhesions $(9,16,27)$. The reduction of adhesions and the perioperative enlargement of the hiatus may increase the risk of $\mathrm{HH}$ after esophagectomy compared to open approaches $(7,9,16,27)$. The outcome of previous reports was in accordance with our results showing that esophagectomies are essentially more frequently associated with $\mathrm{HH}$ compared to total/subtotal gastrectomy (17) and that transhiatal procedures correlated with an even higher incidence due to the enlargement of the hiatus $(9,14)$. In our study, $\mathrm{HH}$ rates after esophagectomy using a transthoracic approach and extended gastrectomy were also significantly higher than after total/subtotal gastrectomy $(2.7 \%$ vs. $6.1 \%$ vs. $0.7 \%$, respectively, $p=0.038$ ). Thus, the surgical techniques should be taken into consideration when evaluating the risk for $\mathrm{HH}$ among surgically treated patients with gastric and esophageal cancer especially in the current era of increased utilization of minimal invasive techniques.

The histological type and stage of the tumor was not significantly associated with the occurrence of $\mathrm{HH}$ in univariate analyses as shown in Table I. This finding is in discordance with a report by Matthews et al. (16) who introduced T-stage as a significant predictor of $\mathrm{HH}$. The majority of oncologic resections in our study were performed in patients with locally advanced esophageal or gastric cancer and correspondingly impaired long-term survival. 
Therefore, late complications such as $\mathrm{HH}$ may be more frequent in studies in which benign disease or more early stage carcinomas are included.

Our data suggested that the presence of preoperative comorbidities, such as diabetes mellitus also significantly affected the development of postoperative $\mathrm{HH}$. In a recent study, the combination of transient neonatal diabetes mellitus (TNDM) and congenital diaphragmatic hernia (CDH) was presented initially, postulating that a common genetic or embryologic etiology may underlie TNDM and CDH (30, 31). Diabetes as a potential predictor for $\mathrm{HH}$ following gastrectomy and esophagectomy has not been previously investigated. However, the correlation between the impairment of wound healing and diabetes $(32,33)$ may have contributed to the increased frequency of $\mathrm{HH}$. Yahchouchy-Chouillard et al. referred to type II diabetes as an important patient-related risk factor for developing incisional hernia following abdominal surgery (34). Further studies reported on the association between diabetes and incisional hernia $(35,36)$, confirming the plausibility of our finding. Finally, Hornby et al. found that diabetes may be a risk factor for recurrent incisional hernia after laparoscopic hernia repair presumably due to impaired wound healing and increased postoperative wound infection complications (37).

Obesity was also found to have an influence on the frequency of $\mathrm{HH}$ in our study and we showed that higher BMI was associated with an increased risk for the development of symptomatic $\mathrm{HH}$ (median BMI with $\mathrm{HH}$ 27 (range $=23-35 \mathrm{~kg} / \mathrm{m}^{2}$ ) vs. BMI without HH 25 $\left(\right.$ range $\left.\left.=15-51 \mathrm{~kg} / \mathrm{m}^{2}\right), p=0.043\right)$. Surprisingly, another clinical study by Ganeshan et al. performed in 2013 indicated opposing results showing that patients with high BMI were less prone to develop $\mathrm{HH}$ after esophagectomy (9). This could be explained by the fact that 'the abdominal contents in patients with BMI $>25 \mathrm{~kg} / \mathrm{m}^{2}$ are increased and therefore may obscure the hiatus" (9). Additionally, Ganeshan et al. considered that less mobility of abdominal contents in patients with high BMI may prevent $\mathrm{HH}$ (9). However, a recent study which assessed the prevalence of $\mathrm{HH}$ in morbidly obese patients based on preoperative upper GI contrast studies, nearly $40 \%$ of patients were identified with $\mathrm{HH}$ (38). Che et al. referred to previous studies showing that obesity is associated with an increased intraabdominal pressure, which may lead to a higher risk of developing abdominal and hiatal herniation (38-41).

Our current retrospective study has some limitations. In this database, different tumor entities were pooled together. However, patients who underwent resection for gastric or esophageal cancer were both included to achieve a representative study size for the investigation of risk factors for the development of $\mathrm{HH}$. Despite the relative small number of patients included, this study is one of the largest from a western comprehensive cancer center providing data regarding potential factors associated with de novo $\mathrm{HH}$ and thus facilitating optimal management of patients at risk.

\section{Conclusion}

De novo $\mathrm{HH}$ is a major adverse event following resection for gastric or esophageal cancer. Among patients undergoing oncological upper GI surgery, extended gastrectomy for carcinoma of the cardia is significantly associated with an increased $\mathrm{HH}$ rate requiring for revisional surgery. The fact that emergent procedures for $\mathrm{HH}$ correlated with high morbidity and mortality underlines the importance of intensive follow-up examinations for high-risk patients and diagnosis of asymptomatic $\mathrm{HH}$ in order to select patients for elective surgery.

\section{Conflicts of Interest}

The Authors report no conflicts of interest relevant to this article.

\section{References}

1 Pennathur A, Gibson MK, Jobe BA and Luketich JD: Oesophageal carcinoma. Lancet 381(9864): 400-412, 2013.

2 Brenkman HJF, Haverkamp L, Ruurda JP and van Hillegersberg $\mathrm{R}$ : Worldwide practice in gastric cancer surgery. World $\mathrm{J}$ Gastroenterol 22(15): 4041-4048, 2016.

3 Xiong JJ, Nunes QM, Huang W, Tan CL, Ke NW, Xie SM, Ran $\mathrm{X}$, Zhang $\mathrm{H}$, Chen $\mathrm{YH}$ and Liu XB: Laparoscopic $v s$. open total gastrectomy for gastric cancer: A meta-analysis. World J Gastroenterol 19(44): 8114-8132, 2013.

4 Gurusamy KS, Pallari E, Midya S and Mughal M: Laparoscopic versus open transhiatal oesophagectomy for oesophageal cancer. Cochrane Database Syst Rev 3: CD011390, 2016.

5 Argenti F, Luhmann A, Dolan R, Wilson M, Podda M, Patil P, Shimi $S$ and Alijani A: Diaphragmatic hernia following oesophagectomy for oesophageal cancer - Are we too radical? Ann Med Surg (Lond) 6: 30-35, 2016.

6 Price TN, Allen MS, Nichols FC, 3rd, Cassivi SD, Wigle DA, Shen KR and Deschamps C: Hiatal hernia after esophagectomy: analysis of 2,182 esophagectomies from a single institution. Ann Thorac Surg 92: 2041-2045, 2011.

7 Kent MS, Luketich JD, Tsai W, Churilla P, Federle M, Landreneau R, Alvelo-Rivera M and Schuchert M: Revisional surgery after esophagectomy: An analysis of 43 patients. Ann Thorac Surg 86: 975-983, 2008.

8 Bronson NW, Luna RA, Hunter JG and Dolan JP: The incidence of hiatal hernia after minimally invasive esophagectomy. J Gastrointest Surg 18: 889-893, 2014.

9 Ganeshan DM, Correa AM, Bhosale P, Vaporciyan AA, Rice D, Mehran RJ, Walsh GL, Iyer R, Roth JA, Swisher SG and Hofstetter WL: Diaphragmatic hernia after esophagectomy in 440 patients with long-term follow-up. Ann Thorac Surg 96: 1138-1145, 2013.

10 Messenger DE, Higgs SM, Dwerryhouse SJ, Hewin DF, Vipond MN, Barr H and Wadley MS: Symptomatic diaphragmatic herniation following open and minimally invasive oesophagectomy: experience from a UK specialist unit. Surg Endosc 29: 417-424, 2015. 
11 Crespin OM, Farjah F, Cuevas C, Armstrong A, Kim BT, Martin AV, Pellegrini CA and Oelschlager BK: Hiatal Herniation After Transhiatal Esophagectomy: an Underreported Complication. J Gastrointest Surg 20(2): 231-236, 2016.

12 Benjamin G, Ashfaq A, Chang YH, Harold K and Jaroszewski D: Diaphragmatic hernia post-minimally invasive esophagectomy: a discussion and review of literature. Hernia 2015 19(4): 635-643, 2015.

13 Sutherland J, Banerji N, Morphew J, Johnson E and Dunn D: Postoperative incidence of incarcerated hiatal hernia and its prevention after robotic transhiatal esophagectomy. Surg Endosc 25(5): 1526-1530, 2011.

14 Gonzalo MA, Almeida $\mathrm{H}$ and Güemes A: Hiatal herniation following total gastrectomy. Rev Esp Enferm Dig 108(4): 234, 2016.

15 Narayanan S, Sanders RL, Herlitz G, Langenfeld J and August DA: Treatment of diaphragmatic hernia occurring after transhiatal esophagectomy. Ann Surg Oncol 22(11): 3681-3686, 2015.

16 Matthews J, Bhanderi S, Mitchell H, Whiting J, Vohra R, Hodson J and Griffiths E: Diaphragmatic herniation following esophagogastric resectional surgery: An increasing problem with minimally invasive techniques? Post-operative diaphragmatic hernias. Surg Endosc 30(12): 5419-5427, 2016.

17 do Nascimento Santos B, Belotto de Oliveira M and D'Alpino Peixotod R: Hiatal Hernia as a Total Gastrectomy Complication. Case Rep Oncol 9(1): 100-105, 2016.

18 Miyagaki H, Takiguchi S, Kurokawa Y, Hirao M, Tamura S, Nishida T, Kimura Y, Fujiwara Y, Mori M and Doki Y: Recent trend of internal hernia occurrence after gastrectomy for gastric cancer. World J Surg 36(4): 851-857, 2012.

19 Murata S, Yamazaki M, Kosugi C, Hirano A, Yoshimura Y, Shiragami R, Suzuki M, Shuto K and Koda K: Hiatal hernia following total gastrectomy with Roux-en-Y reconstruction. Hernia 18(6): 889-891, 2014.

20 Yoshikawa K, Shimada M, Kurita N, Sato H, Iwata T, Higashijima J, Chikakiyo M, Nishi M, Kashihara H, Takasu C, Matsumoto $\mathrm{N}$ and Eto $\mathrm{S}$ : Characteristics of internal hernia after gastrectomy with Roux-en-Y reconstruction for gastric cancer. Surg Endosc 28(6): 1774-1778, 2014.

21 Kojima K, Inokuchi M, Kato K, Motoyama K and Sugihara K: Petersen's hernia after laparoscopic distal gastrectomy with Rouxen-Y reconstruction for gastric cancer. Gastric Cancer 17(1): 146$151,2014$.

22 Larkin JO, Cooke F, Ravi N and Reynolds JV: Internal hernia following total gastrectomy with Roux-en-Y reconstruction. Ann R Coll Surg Engl 93(6): e71-73, 2011.

23 Baba A, Yamazoe S, Dogru M, Okuyama Y, Mogami T, Kobashi Y, Nozawa Y, Aoyagi Y, Fujisaki H, Ogura M and Matsui J: Petersen hernia after open gastrectomy with Roux-en-Y reconstruction: a report of two cases and literature review. SpringerPlus 4: 753, 2015.

24 Tashiro Y, Murakami M, Otsuka K, Saito K, Saito A, Motegi K, Date H, Yamashita T, Ariyoshi T, Goto S, Yamazaki K, Fujimori A, Watanabe $M$ and Aoki T: Intrathoracic Hernia after Total Gastrectomy. Case Rep Gastroenterol 10(1): 1-6, 2016.

25 Santos Bdo N, de Oliveira MB and Peixoto RD: Hiatal Hernia as a Total Gastrectomy Complication. Case Rep Oncol 9(1): 100-105, 2016.

26 Piciucchi S, Milandri C, Verdecchia GM, Framarini M, Amadori E, Monti M, Oboldi D, Bandi G, Barone D and Gavelli G: Acute hiatal hernia: a late complication following gastrectomy. Int Arch Med 3: 23, 2010.
27 Vallböhmer D, Hölscher AH, Herbold T, Gutschow C and Schröder W: Diaphragmatic hernia after conventional or laparoscopic-assisted transthoracic esophagectomy. Ann Thorac Surg 84(6): 1847-1852, 2017.

28 Andreou A, Biebl M, Dadras M, Struecker B, Sauer IM, ThussPatience PC, Chopra S, Fikatas P, Bahra M, Seehofer D, Pratschke $\mathrm{J}$ and Schmidt SC: Anastomotic leak predicts diminished long-term survival after resection for gastric and esophageal cancer. Surgery 160(1): 191-203, 2016.

29 van Sandick JW, Knegjens JL, van Lanschot JJ and Obertop H: Diaphragmatic herniation following oesophagectomy. Br J Surg 86(1): 109-112, 1999.

30 Topiol ES, Minarich LA, Williams CA, Zori RT, Kays DW and Haller MJ: Neonatal diabetes mellitus and congenital diaphragmatic hernia: coincidence or concurrent etiology? Int $\mathrm{J}$ Pediatr Endocrinol 2012(1): 21, 2012.

31 Gortner L: Manifestation of a congenital diaphragmatic hernia and molecular pathogenesis. Klin Padiatr 224(7): 429-430, 2012.

32 Falanga V: Wound healing and its impairment in the diabetic foot. Lancet 2005 366(9498): 1736-1743, 2005.

33 Dangwal S, Stratmann B, Bang C, Lorenzen JM, Kumarswamy R, Fiedler J, Falk CS, Scholz CJ, Thum T and Tschoepe D: Impairment of Wound Healing in Patients With Type 2 Diabetes Mellitus Influences Circulating MicroRNA Patterns via Inflammatory Cytokines. Arterioscler Thromb Vasc Biol 35(6): 1480-1488, 2015.

34 Yahchouchy-Chouillard EK, Aura T, Picone O, Etienne JC and Fingerhut A: Incisional Hernias. Dig Surg 20(1): 3-9, 2003.

35 Scheuerlein H, Settmacher U, Lenschow M and Rauchfuss F: Complex Incisional Hernias. Arch Clin Gastroenterol 2(1): 017026, 2016.

36 Dietz UA, Winkler MS, Härtel RW, Fleischhacker A, Wiegering $\mathrm{A}$, Isbert $\mathrm{C}$, Jurowich $\mathrm{Ch}$, Heuschmann $\mathrm{P}$ and Germer CT: Importance of recurrence rating, morphology, hernial gap size, and risk factors in ventral and incisional hernia classification. Hernia 18(1): 19-30, 2014.

37 Hornby ST, McDermott FD, Coleman M, Ahmed Z, Bunni J, Bunting D, Elshaer M, Evans V, Kimble A, Kostalas M, Page G, Singh J, Szczebiot L, Wienand-Barnett S, Wilkins A, Williams $\mathrm{O}$ and Newell P: Female gender and diabetes mellitus increase the risk of recurrence after laparoscopic incisional hernia repair. Ann R Coll Surg Engl 97(2): 115-119, 2015.

38 Che F, Nguyen B, Cohen A and Nguyen NT: Prevalence of hiatal hernia in the morbidly obese. Surg Obes Relat Dis 9(6): 920-924, 2013.

39 Sonnenberg A: Effects of environment and lifestyle on gastroesophageal reflux disease. Dig Dis 29: 229-234, 2011.

40 Varela JE, Hinojosa M and Nguyen N: Correlations between intra-abdominal pressure and obesity-related co-morbidities. Surg Obes Relat Dis 5(5): 524-528, 2009.

41 Wilson LJ, Ma W and Hirschowitz BI: Association of obesity with hiatal hernia and esophagitis. Am J Gastroenterol 94: 2840, 1999.

Received September 12, 2017

Revised October 8, 2017

Accepted October 12, 2017 